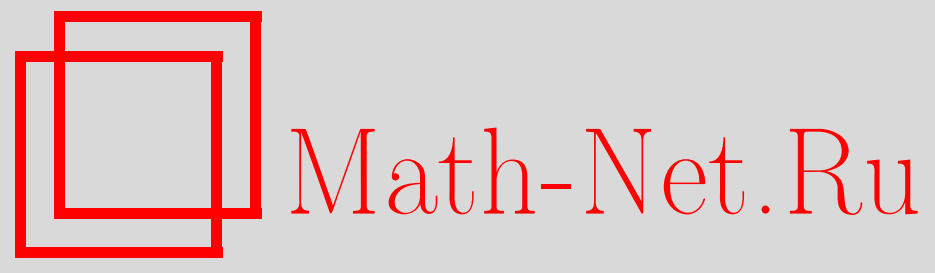

Л. В. Степанова, Е. М. Яковлева, О смешанном нагружении элементов конструкции с дефектом, Вестн. Сам. гос. техн. ун-та. Сер. Физ.-мат. науки, 2015, номер 2, 358-381

DOI: https://doi.org/10.14498/vsgtu1432

Использование Общероссийского математического портала MathNet.Ru подразумевает, что вы прочитали и согласны с пользовательским соглашением

http://www . mathnet.ru/rus/agreement

Параметры загрузки:

IP : 54.162 .85 .209

26 апреля 2023 г., 09:52:22

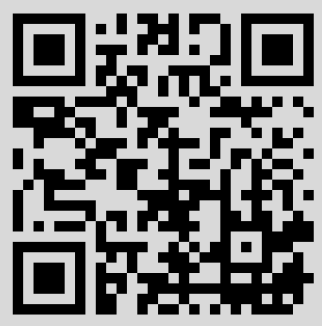




\title{
О СМЕШАННОМ НАГРУЖЕНИИ ЭЛЕМЕНТОВ КОНСТРУКЦИИ С ДЕФЕКТОМ*
}

\section{Л. В. Степанова, Е. М. Яковлева}

Самарский государственный университет,

Россия, 443011, Самара, ул. Академика Павлова, 1.

\begin{abstract}
Аннотация
В статье рассматривается задача определения напряженно-деформированного состояния в окрестности вершины трещины в случае смешанного нагружения (нормальный отрыв и поперечный сдвиг) в полном диапазоне смешанных форм деформирования в условиях плоского напряженного состояния. Для построения асимптотического решения используется метод разложения по собственным функциям механических полей в окрестности вершины трещины. Показано, что проблема отыскания полей напряжений и деформаций в окрестности вершины трещины сводится к нелинейной задаче на собственные значения, где показатель степени расстояния от вершины трещины представляет собой собственное значение, а угловое распределение компонент тензора напряжений - собственные функции. Получено численное решение нелинейной задачи на собственные значения и найден весь спектр собственных значений. Найдены новые собственные значения, отличные от собственных значений классической задачи Хатчинсона-Райса-Розенгрена. Показано, что новое асимптотическое решение можно интерпретировать как автомодельно-промежуточную асимптотику поля напряжений на расстояниях, много меньших характерного линейного размера образца, но много больших характерного линейного размера области полностью поврежденного материала.
\end{abstract}

Ключевые слова: нелинейная задача на собственные значения, напряженно-деформированное состояние у вершины трещины, смешанное деформирование, параметр смешанности, метод разложения по собственным функциям.

doi: http://dx.doi.org/10.14498/vsgtu1432

(C) 2015 Самарский государственный технический университет.

\section{Образец для цитирования}

С теп анов а Л. В., Я ков лев а Е. М. О смешанном нагружении элементов конструкции с дефектом // Вестн. Сам. гос. техн. ун-та. Сер. Физ.-мат. науки, 2015. Т. 19, № 2. C. 358-381. doi: 10.14498/vsgtu1432.

\section{Сведения об авторах}

Лариса Валентиновна Степанова (д.ф.-м.н., доцент; stepanovalv@samsu.ru; автор, ведущий переписку), профессор, каф. математического моделирования в механике.

Екатерина Михайловна Яковлева (adylinaem@samsu.ru), аспирант, каф. математического моделирования в механике.

*Настоящая статья представляет собой расширенный вариант доклада [1], сделанного авторами на Четвёртой международной конференции «Математическая физика и её приложения» (Россия, Самара, 25 августа - 1 сентября 2014). 
Введение. О смешанном деформировании элементов конструкций с трещинами. Асимптотический анализ распределений напряжений, деформаций (или скоростей деформаций) и перемещений вблизи вершины трещины является одной из фундаментальных задач механики трещин и механики поврежденности [2]. Вершина трещины может находиться в упругом материале, пластической зоне или зоне, занятой деформациями ползучести; кончик трещины может принадлежать линии раздела нескольких изотропных или анизотропных материалов [3]. Все перечисленные факторы усложняют определение механических полей вблизи устья трещины и до настоящего времени многие вопросы, связанные с нахождением напряженно-деформированного состояния в нелинейной механике разрушения, остаются открытыми. Так, в настоящее время в механике трещин и в целом в механике разрушения сложилось понимание процесса разрушения как процесса многоскейлингового (многомасштабного, многоуровневого), для описания основных закономерностей которого следует прибегать к многомасштабным моделям [4-8]. В рамках многоуровневого подхода процесс разрушения моделируется различными соотношениями на различных расстояниях от вершины трещины с помощью введения многих масштабов. При построении решения задачи в целом представления, работающие на разных расстояниях от кончика трещины, сращиваются в зонах, где справедливы асимптотики соседних областей. Для понимания многоуровневого или многомасштабного подхода целесообразно обратиться к исходным математическим моделям, используемым как в теории механики трещин, так и в инженерной практике $[9,10]$. В рамках линейной теории упругости с помощью методов теории возмущений построено большое количество решений, ставших классическими в механике хрупкого разрушения $[11,12]$. Однако для нелинейной механики многие вопросы остаются открытыми $[10,13]$.

Определение напряженно-деформированного состояния вблизи кончика трещины в материале со степенным законом упрочнения с помощью асимптотических разложений полей напряжений и деформаций в окрестности вершины трещины и построение высших приближений асимптотических разложений являлись предметом многочисленных исследований на протяжении многих лет, начиная с классических работ Дж. Хатчинсона, Дж. Райса и Дж. Розенгрена (HRR) [13-16] до работ самого последнего времени [17-20].

Поскольку элементы ответственных конструкций, находящихся в реальных эксплуатационных условиях, подвержены действию сложных систем механических нагрузок, характерной для них является эксплуатация в условиях сложного напряженного состояния при наличии различным образом ориентированных дефектов. Поэтому понятен интерес специалистов к задачам о наклонных трещинах и о трещинах, находящихся в условиях одновременно приложенных нормального растягивающего напряжения и поперечного сдвига, которые в механике разрушения относятся к классу смешанных мод нагружения.

В классической механике разрушения принято выделять три вида трещин, отвечающих трем видам нагружения: трещины нормального отрыва, поперечного и продольного (антиплоского) сдвига. Выделение трех типов трещин восходит к линейной механике разрушения $[11,12]$, где суперпозиция решений, полученных для трех типов нагружения, дает решение, спра- 
ведливое вблизи вершины трещины, для произвольного нагружения тела с дефектом. Указанные типы нагружения элемента конструкции с трещинами в линейно упругих материалах, в идеально пластических телах достаточно хорошо изучены. Однако распределение напряжений, деформаций и перемещений вблизи кончика дефекта в условиях смешанного нагружения трещины в нелинейных материалах изучено недостаточно [10, 13, 21].

Можно отметить, что первое обращение к исследованию смешанных форм деформирования, по всей видимости, было сделано в работах Ши [22, 23], где изучены поля напряжений и деформаций вблизи вершины трещины, находящейся под действием растягивающей и сдвиговой нагрузок (т.о. рассматривалось смешанное нагружение, отвечающее трещинам типа I и типа II). Ши впервые ввел коэффициент смешанности нагружения [22]:

$$
M^{p}=\frac{2}{\pi} \operatorname{arctg}\left|\lim _{r \rightarrow 0} \frac{\sigma_{\theta \theta}(r, \theta=0)}{\sigma_{r \theta}(r, \theta=0)}\right|,
$$

принимающих нулевое значение для трещины поперечного сдвига; значение, равное единице, для чистого растяжения и значение $0<M^{p}<1$ для смешанных форм нагружения образца с трещиной. С тех пор анализ смешанных мод деформирования является объектом пристального внимания ученых как в России [24-26], так и за рубежом [27,28]. В [25] разработан метод и приведены результаты расчетов упругих параметров смешанности и скорости выделения энергии в трехмерной постановке при сочетании форм смещения поверхностей трещины по типу отрыва, сдвига и среза. Рассмотрено нагружение произвольно ориентированной несквозной полуэллиптической трещины при двухосном нагружении различной интенсивности. Алгоритм вычислений построен на модификации аналитического решения с помощью корректирующих функций учета выхода вершин полуэллиптической трещины на свободную поверхность пластины. На основе проведенного анализа и выполненных расчетов установлен характер влияния вида смешанных форм нагружения и формы в плане несквозного дефекта на изменение параметров вдоль криволинейного фронта трещины. В [26] изложен метод и приведены результаты расчетов упругопластических коэффициентов интенсивности напряжений в полном диапазоне смешанных форм деформирования от нормального отрыва до чистого сдвига. Рассмотрено состояние произвольно ориентированной прямолинейной трещины в виде математического разреза при двухосном нагружении различной интенсивности. Решение построено на использовании уравнения совместности деформаций, представленного через функцию напряжений Эри и ее производные. Поведение упругопластического материала соответствует модели Рамберга-Осгуда. На основе выполненных расчетов установлен характер влияния вида смешанных форм нагружения и пластических свойств материала, описываемых показателем деформационного упрочнения.

В настоящее время сложилось понимание необходимости определения всего спектра собственных значений в задаче HRR. В линейной механике разрушения в качестве базового распределения может быть выбрано классическое решение М. Уильямса $[11,12]$ с корневой особенностью. Более сильная или более слабая особенности могут быть введены для моделирования степени поврежденности (степени разрушения) материала у кончика трещины на 
уровне мезо- и микроструктуры материала. Математически данная модель приводит к необходимости всего спектра собственных значений в задачах на собственные значения, следующих из проблем нахождения напряженнодеформированного состояния у окрестности вершины трещины. Если в линейной механике разрушения весь спектр собственных значений найден и построено полное асимптотическое представление полей напряжений и перемещений (решение М. Уильямса $[11,12])$, то в нелинейной механике разрушения остается открытым вопрос о спектре собственных значений нелинейной задачи на собственные значения, следующей из проблемы отыскания полей напряжений у вершины трещины в материалах со степенными определяющими уравнениями.

Совокупность нелинейных задач на собственные значения, возникающих в нелинейной механике разрушения, представляется важной в связи с необходимостью применения многомасштабных, многоуровневых моделей [29-31], в соответствии с которыми в окрестности вершины трещины необходимо вводить в рассмотрение иерархию областей с доминирующим действием различных асимптотик поля напряжений и проводить процедуру асимптотического сращивания получаемых ассимптотических решений. Так, в работе [31] построено новое асимптотическое решение задачи об усталостном росте трещины в изотропном линейно-упругом материале с учетом процесса накопления рассеянных повреждений в рамках связанной (упругость-поврежденность) постановки задачи в условиях реализации плоского напряженного и плоского деформированного состояния. В [31] представлено новое численное решение двухточечной краевой задачи для системы обыкновенных дифференциальных уравнений, которая следует из проблемы определения напряженнодеформированного состояния у кончика трещины в среде с поврежденностью, а также новое аналитическое представление полей напряжений, деформаций и сплошности в окрестности вершины трещины как для плоского напряженного, так и для плоского деформированного состояний. Аккуратное построение всех промежуточных зон с той или иной асимптотикой и проведение процедуры сращивания требует знания всего спектра собственных значений, и, по всей видимости, эти задачи до сих пор не решены. В литературе известны только две математически полностью реализованные процедуры сращивания: классическое решение Райса-Нейбери для трещины антиплоского сдвига [32] и аналитическое решение задачи о трещине конечной длины в бесконечной пластине [21].

Настоящая работа является продолжением исследования [33], где представлен численный метод определения спектра собственных значений в нелинейной задаче на собственные значения, следующей из проблемы определения напряженно-деформированного состояния в окрестности вершины трещины в условиях смешанного деформирования для материала со степенными определяющими уравнениями (степенной закон деформационной теории пластичности, степенной закон установившейся ползучести) в условиях плоского напряженного состояния. В [33] найдено, что смешанное нагружение пластины с дефектом приводит к изменению особенности поля напряжений вблизи кончика трещины, и к решению, отличному от классического решения Хатчинсона-Райса-Розенгрена. Предлагаемый метод использован в данной работе для нахождения всего спектра собственных чисел, отличных от найденных ранее. 
Построены угловые распределения компонент тензора напряжений (собственные функции) в полном диапазоне значений параметра смешанности нагружения от чистого нормального отрыва до поперечного сдвига.

\section{1. Задача определения напряженно-деформированного состояния вблизи} кончика трещины в материале со степенным определяющим законом. Исследование напряженно-деформированного состояния в окрестности вершины трещины нормального отрыва в материале со степенными определяющими уравнениями

$$
\varepsilon_{i j}=3 B \sigma_{e}^{n-1} s_{i j} / 2,
$$

где $\varepsilon_{i j}$ - компоненты тензора деформаций; $s_{i j}$ - компоненты девиатора тензора напряжений; $\sigma_{e}=\left(3 s_{i j} s_{i j} / 2\right)^{1 / 2}$ - интенсивность напряжений; $B, n-$ материальные константы, определяемые экспериментально, в полярных координатах с полюсом в берегах трещины, приводит к необходимости исследования уравнений равновесия

$$
\frac{\partial \sigma_{r r}}{\partial r}+\frac{1}{r} \frac{\partial \sigma_{r \theta}}{\partial \theta}+\frac{\sigma_{r r}-\sigma_{\theta \theta}}{r}=0, \quad \frac{\partial \sigma_{r \theta}}{\partial r}+\frac{1}{r} \frac{\partial \sigma_{\theta \theta}}{\partial \theta}+2 \frac{\sigma_{r \theta}}{r}=0
$$

и условия совместности деформаций

$$
2 \frac{\partial}{\partial r}\left(r \frac{\partial \varepsilon_{r \theta}}{\partial \theta}\right)=\frac{\partial^{2} \varepsilon_{r r}}{\partial \theta^{2}}-r \frac{\partial \varepsilon_{r r}}{\partial r}+r \frac{\partial^{2}\left(r \varepsilon_{\theta \theta}\right)}{\partial r^{2}} .
$$

В случае плоского напряженного состояния определяющие соотношения материала (2) принимают вид

$$
\varepsilon_{r r}=\frac{1}{2} B \sigma_{e}^{n-1}\left(2 \sigma_{r r}-\sigma_{\theta \theta}\right), \quad \varepsilon_{\theta \theta}=\frac{1}{2} B \sigma_{e}^{n-1}\left(2 \sigma_{\theta \theta}-\sigma_{r r}\right), \quad \varepsilon_{r \theta}=\frac{3}{2} B \sigma_{e}^{n-1} \sigma_{r \theta},
$$

где интенсивность касательных напряжений определяется формулой

$$
\sigma_{e}=\sqrt{\sigma_{r r}^{2}+\sigma_{\theta \theta}^{2}-\sigma_{r r} \sigma_{\theta \theta}+3 \sigma_{r \theta}^{2}}
$$

Степенной характер определяющих уравнений задачи (2) позволяет обратиться к представлению функции напряжений Эри в виде разложения по собственным функциям при $r \rightarrow 0$ :

$$
F(r, \theta)=r^{\lambda+1} f(\theta) .
$$

Тогда компоненты тензора деформации принимают вид

$$
\begin{aligned}
& \varepsilon_{r r}(r, \theta)=\frac{1}{2} B r^{(\lambda-1) n} f_{e}^{n-1}\left\{\left[2(\lambda+1) f(\theta)+f^{\prime \prime}(\theta)\right]-(\lambda+1) \lambda f(\theta)\right\}, \\
& \varepsilon_{\theta \theta}(r, \theta)=\frac{1}{2} B r^{(\lambda-1) n} f_{e}^{n-1}\left[(\lambda+1)(2 \lambda-1) f(\theta)-f^{\prime \prime}(\theta)\right], \\
& \varepsilon_{r \theta}(r, \theta)=-\frac{3}{2} B r^{(\lambda-1) n} f_{e}^{n-1} \lambda f^{\prime}(\theta),
\end{aligned}
$$

где принято обозначение

$$
\begin{aligned}
f_{e}^{2}=\left[(\lambda+1) f(\theta)+f^{\prime \prime}(\theta)\right]^{2} & +(\lambda+1)^{2} \lambda^{2} f^{2}(\theta)- \\
& -\left[(\lambda+1) f(\theta)+f^{\prime \prime}(\theta)\right](\lambda+1) \lambda f(\theta)+3 \lambda^{2} f^{2}(\theta) .
\end{aligned}
$$


Условие совместности деформаций приводит к нелинейному обыкновенному дифференциальному уравнению (ОДУ) четвертого порядка относительно функции $f(\theta)$ :

$$
\begin{aligned}
& f^{I V} f_{e}^{2}\left[(n-1) x\left[y-\frac{1}{2} \lambda(\lambda+1) f\right]+2 f\right]+6 \lambda[(\lambda-1) n+1]\left[(n-1) f_{e}^{2} g f^{\prime}+f_{e}^{4} f^{\prime \prime}\right]+ \\
& +(n-1)(n-3) g^{2} x+(n-1) f_{e}^{2} x \omega+2(n+1) f_{e}^{2} g x^{\prime}+f_{e}^{4}\left[(\lambda+1)(2-\lambda) f^{\prime \prime}\right]- \\
& \quad-(\lambda-1) n f_{e}^{4} x+[(\lambda-1) n+1](\lambda-1) n f_{e}^{4}\left[(\lambda+1)(2 \lambda-1) f-f^{\prime \prime}\right]=0, \quad(5)
\end{aligned}
$$

где приняты следующие сокращающие обозначения:

$$
\begin{gathered}
y=(\lambda+1) f+f^{\prime \prime}, \quad x=(\lambda+1)(\lambda-2) f+f^{\prime \prime}, \\
f_{e}^{2}=y^{2}+\lambda^{2}(\lambda+1)^{2} f^{2}-y \lambda(\lambda+1) f+3 \lambda^{2} f^{\prime 2}, \\
g=y y^{\prime}+\lambda^{2}(\lambda+1)^{2} f f^{\prime}-\lambda(\lambda+1) f^{\prime} y / 2-\lambda(\lambda+1) f y^{\prime} / 2+3 \lambda^{2} f^{\prime} f^{\prime \prime}, \\
\omega=y^{\prime 2}+y(\lambda+1) f^{\prime \prime}+\lambda^{2}(\lambda+1)^{2}\left[f^{\prime 2}+f^{\prime} f^{\prime \prime}\right]-\lambda(\lambda+1) f^{\prime \prime} y / 2- \\
-\lambda(\lambda+1) f^{\prime} y^{\prime}-\lambda(\lambda+1)^{2} f f^{\prime \prime} / 2+3 \lambda^{2}\left[f^{\prime \prime 2}+f^{\prime} f^{\prime \prime \prime}\right] .
\end{gathered}
$$

Решение нелинейного однородного дифференциального уравнения четвертого порядка (5) должно удовлетворять краевым условиям, следующим из требования отсутствия поверхностных усилий на берегах трещины:

$$
f(\theta= \pm \pi)=0, \quad f^{\prime}(\theta= \pm \pi)=0 .
$$

ОДУ (5) вместе с краевыми условиями (6) представляет собой двухточечную краевую задачу на собственные значения: необходимо найти собственные значения $\lambda$, отвечающие нетривиальным решениям уравнения (5), удовлетворяющим краевым условиям (6). Для решения такого ряда задач обычно обращаются к численным методам: семейству методов Рунге-Кутта-Фельберга и методу пристрелки, в рамках которых первоначально необходимо перейти к задаче Коши для уравнения (5).

В механике разрушения выделяют три вида нагружения образца с дефектом: нормальный отрыв (тип I), поперечный сдвиг (тип II) и продольный (антиплоский) сдвиг (тип III).

Симметричное раскрытие берегов трещины (трещины типа I) приводит к следующим начальным условиям:

$$
f(\theta=0)=1, \quad f^{\prime}(\theta=0)=0, \quad f^{\prime \prime}(\theta=0)=A_{2}, \quad f^{\prime \prime \prime}(\theta=0)=0 .
$$

Уравнение (5) вместе с начальными условиями (7) интегрируется численно. В случае трещины нормального отрыва находят два значения: $\lambda$ и $A_{2}$, таким образом, чтобы выполнялись краевые условия на верхнем берегу дефекта

$$
f(\theta=\pi)=0, \quad f^{\prime}(\theta=\pi)=0 .
$$

Для трещины типа II (антисимметричное деформирование) начальные условия имеют вид

$$
f(\theta=0)=0, \quad f^{\prime}(\theta=0)=1, \quad f^{\prime \prime}(\theta=0)=0, \quad f^{\prime \prime \prime}(\theta=0)=A_{3}
$$

и для выполнения условий отсутствия поверхностных усилий подбираются значения параметров $\lambda$ и $A_{3}$. 
Одно собственное значение хорошо известно и соответствует классической задаче Хатчинсона-Райса-Розенгрена (HRR) [14-16]: $\lambda=n /(n+1)$. Однако сейчас интерес представляют другие собственные значения, отличные от собственных чисел, отвечающих задаче $\operatorname{HRR}[10,29,30]$.

2. Численный алгоритм определения собственных значений. В случае смешанного деформирования соображения симметрии и антисимметрии использованы быть не могут и необходимо искать решение уравнения (5) на отрезке $[-\pi, \pi]$. В условиях смешанного нагружения при численном решении уравнения (5) отрезок интегрирования $[-\pi, \pi]$ можно разбить на два отрезка: $[-\pi, 0]$ и $[0, \pi]$. Сначала уравнение $(5)$ интегрируется на отрезке $[0, \pi]$ и краевая задача сводится к задаче Коши с начальными условиями

$$
\begin{gathered}
f(\theta=0)=1, \quad f^{\prime}(\theta=0)=-(\lambda+1) / \operatorname{tg}\left(M^{p} \pi / 2\right), \\
f^{\prime \prime}(\theta=0)=A_{2}, \quad f^{\prime \prime \prime}(\theta=0)=A_{3} .
\end{gathered}
$$

Значение производной первого порядка находится из условия смешанности нагружения (значение параметра смешанности нагружения (1), задающего вид нагружения, известно). Неизвестные постоянные $A_{2}$ и $A_{3}$ определяются таким образом, чтобы выполнялись краевые условия на верхнем берегу трещины:

$$
f(\theta=\pi)=0, \quad f^{\prime}(\theta=\pi)=0 .
$$

После того как подобраны постоянные $A_{2}$ и $A_{3}$, уравнение (5) интегрируется на отрезке $[-\pi, 0]$. Вновь двухточечная краевая задача для уравнения (5) с граничными условиями

$$
\begin{gathered}
f(\theta=-\pi)=0, \quad f^{\prime}(\theta=-\pi)=0, \quad f^{\prime \prime}(\theta=-\pi)=1 \\
f^{\prime \prime \prime}(\theta=-\pi)=-(\lambda+1) / \operatorname{tg}\left(M^{p} \pi / 2\right)
\end{gathered}
$$

заменяется задачей Коши с начальными условиями

$$
f(\theta=-\pi)=0, \quad f^{\prime}(\theta=-\pi)=0, \quad f^{\prime \prime}(\theta=-\pi)=B_{2}, \quad f^{\prime \prime \prime}(\theta=-\pi)=B_{3} .
$$

Неизвестные постоянные $B_{2}$ и $B_{3}$ подбираются таким образом, чтобы выполнялись условия равновесия элемента, расположенного на луче $\theta=0$. Уравнения равновесия данного элемента требуют непрерывности компонент тензора напряжений $\sigma_{\theta \theta}$ и $\sigma_{r \theta}$ на луче $\theta=0$, что влечет за собой непрерывность функций $f(\theta)$ и $f^{\prime}(\theta)$ при $\theta=0$ (и, следовательно, краевые условия $(8))$. Поэтому две неизвестные постоянные $B_{2}$ и $B_{3}$ определяются таким образом, чтобы решение, разыскиваемое на отрезке $[-\pi, 0]$, удовлетворяло краевым условиям при $\theta=0$. Более подробно процедура метода описана в [33]. Предложенный ниже метод используется для отыскания спектра собственных чисел.

При построении новых собственных значений накладывалось дополнительное условие - требование непрерывности компоненты тензора напряжений. Результаты вычислений приведены в табл. 1-4, где собраны новые значения $\lambda$ и пристрелочные значения

$$
f^{\prime \prime}(\theta=0), \quad f^{\prime \prime \prime}(\theta=0), \quad f^{\prime \prime}(\theta=-\pi) \quad \text { и } \quad f^{\prime \prime \prime}(\theta=-\pi)
$$


для всех значений параметра смешанности нагружения и практически важных значений показателя нелинейности материала $M^{p}$.

Угловые распределения компонент тензора напряжений $\sigma_{i j}$ для различных значений параметра смешанности нагружения $M^{p}$ и показателя нелинейности материала $n=6$ показаны на рис. $1-3$, а для $n=8-$ на рис. 4-6.

3. Автомодельное решение задачи о трещине в среде с поврежденностью в условиях смешанного деформирования. Промежуточная автомодельная асимптотика. Рассмотрим приложение найденной новой асимптотики поля напряжений к задаче о стационарной трещине в среде с поврежденностью в условиях смешанного нагружения и построим автомодельное промежуточное асимптотическое решение задачи о трещине в связанной (ползучесть поврежденность) постановке задачи.

Определяющие уравнения материала строятся на основе степенного закона Бейли-Нортона теории установившейся ползучести с применением концепции эффективного напряжения $[4,34]$ :

$$
\dot{\varepsilon}_{i j}=\frac{3}{2} B\left(\frac{\sigma_{e}}{\psi}\right)^{n-1} \frac{s_{i j}}{\psi} .
$$

где $\psi$ - параметр сплошности, эволюционирующий в соответствии со степенным законом накопления повреждений

$$
\frac{d \psi}{d t}=-A\left(\frac{\sigma_{e}}{\psi}\right)^{m}
$$

Естественно предположить, что процесс активного накопления рассеянных повреждений происходит в непосредственной окрестности вершины трещины, где образуется область диспергированного (дефрагментированного) материала, а на удалении от нее параметр сплошности стремится к единице, что отвечает неповрежденному материалу. Данное предположение позволяет сформулировать асимптотическое граничное условие в бесконечно удаленной точке как условие асимптотического сближения с решением ХатчинсонаРайса-Розенгрена (HRR) - решением для степенных определяющих уравнений $(9)$, где $\psi \equiv 1$ (таким образом, рассматривается непосредственная окрестность вершины трещины). В соответствии с гипотезой о маломасштабной поврежденности на больших расстояниях от вершины трещины (больших по сравнению с характерным линейным размером области полностью поврежденного материала, но все еще малых по сравнению с длиной трещины, характерным линейным размером образца) после напряжений определяется решением HRR (решением аналогичной задачи без учета процесса накопления повреждений $\psi \equiv 1)$ :

$$
\sigma_{i j}(r \rightarrow \infty, \theta, t)=\left(\frac{C^{*}}{B I_{n} r}\right)^{1 /(n+1)} \overline{\sigma_{i j}}(\theta, n) .
$$

Начальное условие при $t=0$ и граничное условие в бесконечно удаленной точке (11) совпадают, поскольку они задаются решением задачи для $\psi \equiv 1$.

Анализ размерности величин, входящих в уравнения (9)-(11), позволяет установить, что для определяющих соотношений (9), кинетического уравнения (10), начальных и граничных условий (11) существует автомодельная 
Таблица 1

Собственные значения для различных значений параметра смешанности нагружения в условиях плоского напряженного состояния $n=2$ [Eigenvalues for different values of the mixity parameter. Plane stress conditions; $n=2$ ]

\begin{tabular}{cccccc}
\hline$M^{p}$ & $\lambda$ & $f^{\prime \prime}(0)$ & $f^{\prime \prime \prime}(0)$ & $f^{\prime \prime}(-\pi)$ & $f^{\prime \prime \prime}(-\pi)$ \\
\hline 0.95 & -0.30224000 & -0.24670803 & -0.45261423 & 0.46460644 & 0.31524251 \\
0.90 & -0.30032000 & -0.25428500 & -0.52319280 & 0.36781000 & 0.41793000 \\
0.80 & -0.28609000 & -0.30988600 & -0.65543910 & -0.14222000 & 1.23657500 \\
0.70 & -0.26789000 & -0.40297913 & -0.80444475 & -0.37921000 & 0.54939150 \\
0.60 & -0.26093000 & -0.46493199 & -1.03847110 & -0.54340000 & 0.46094200 \\
0.50 & -0.25233200 & -0.52217930 & -1.40075019 & -0.72780000 & 0.42459230 \\
0.40 & -0.24369800 & -0.57136233 & -1.98711539 & -0.97155000 & 0.40989380 \\
0.30 & -0.23701900 & -0.61089207 & -2.95625279 & -1.35116000 & 0.41294900 \\
0.20 & -0.23247900 & -0.64000914 & -4.76598544 & -2.08610169 & 0.44422935 \\
0.10 & -0.22987230 & -0.65774480 & -9.82544937 & -4.26300089 & 0.57184713 \\
0.05 & -0.22923480 & -0.66219933 & -19.65958382 & -8.60607626 & 0.84616996 \\
\hline
\end{tabular}

Таблица 2

Собственные значения для различных значений параметра смешанности нагружения в условиях плоского напряженного состояния $n=4$ [Eigenvalues for different values of the mixity parameter. Plane stress conditions; $n=4]$

\begin{tabular}{ccccrr}
\hline$M^{p}$ & $\lambda$ & $f^{\prime \prime}(0)$ & $f^{\prime \prime \prime}(0)$ & $f^{\prime \prime}(-\pi)$ & \multicolumn{1}{c}{$f^{\prime \prime \prime}(-\pi)$} \\
\hline 0.95 & -0.25900000 & 0.06403000 & -0.76644890 & 1.00747157 & -0.49344443 \\
0.90 & -0.25560000 & 0.06293000 & -0.84040608 & 0.91827825 & -0.44735992 \\
0.80 & -0.24450000 & 0.06180000 & -0.99744294 & 0.72118794 & -0.34292779 \\
0.70 & -0.23350000 & 0.06296000 & -1.18109399 & 0.47043046 & -0.19390281 \\
0.60 & -0.22020000 & 0.02702000 & -1.38664305 & -0.26870000 & 0.33821000 \\
0.50 & -0.21079000 & 0.02508000 & -1.68869370 & -0.45820000 & 0.27883500 \\
0.40 & -0.20527000 & 0.07940000 & -2.16329580 & -0.70405000 & 0.36147500 \\
0.30 & -0.20303000 & 0.19626828 & -2.96732416 & -1.10930000 & 0.53612990 \\
0.20 & -0.20573000 & 0.41709000 & -4.60578260 & -1.93328000 & 0.91471369 \\
0.10 & -0.21570000 & 0.89713000 & -9.66506720 & -4.50572000 & 2.13143060 \\
0.05 & -0.22414000 & 1.42971500 & -19.75640850 & -9.85286900 & 4.68905308 \\
\hline
\end{tabular}

Таблица 3

Собственные значения для различных значений параметра смешанности нагружения в условиях плоского напряженного состояния $n=6$ [Eigenvalues for different values of the mixity parameter. Plane stress conditions; $n=6$ ]

\begin{tabular}{ccccrr}
\hline$M^{p}$ & $\lambda$ & $f^{\prime \prime}(0)$ & $f^{\prime \prime \prime}(0)$ & $f^{\prime \prime}(-\pi)$ & $f^{\prime \prime \prime}(-\pi)$ \\
\hline 0.95 & -0.23620000 & 0.12763000 & -0.88589036 & 1.10948713 & -0.69773171 \\
0.90 & -0.23000000 & 0.12630000 & -0.95970043 & 1.00961648 & -0.63151712 \\
0.80 & -0.21800000 & 0.13638000 & -1.12682114 & 0.81269900 & -0.50297035 \\
0.70 & -0.20880000 & 0.15539000 & -1.32590338 & 0.58885377 & -0.36077682 \\
0.60 & -0.19930000 & 0.15484000 & -1.55328928 & -0.16700000 & 0.69325000 \\
0.50 & -0.19078000 & 0.15920000 & -1.85600631 & -0.37120000 & 0.23952300 \\
0.40 & -0.18870000 & 0.24360000 & -2.34861936 & -0.61155000 & 0.37189580 \\
0.30 & -0.19332000 & 0.40705000 & -3.19053122 & -1.01899000 & 0.61801287 \\
0.20 & -0.20619500 & 0.70100000 & -4.94411518 & -1.86793000 & 1.14461341 \\
0.10 & -0.23041870 & 1.34989000 & -10.72665207 & -4.59496000 & 2.87542559 \\
0.05 & -0.24985460 & 2.15447000 & -23.20019415 & -10.42638000 & 6.63434136 \\
\hline
\end{tabular}


Таблица 4

Собственные значения для различных значений параметра смешанности нагружения в условиях плоского напряженного состояния $n=8$ [Eigenvalues for different values of the mixity parameter. Plane stress conditions; $\boldsymbol{n}=8]$

\begin{tabular}{ccccrr}
\hline$M^{p}$ & $\lambda$ & $f^{\prime \prime}(0)$ & $f^{\prime \prime \prime}(0)$ & $f^{\prime \prime}(-\pi)$ & $f^{\prime \prime \prime}(-\pi)$ \\
\hline 0.95 & -0.22400000 & 0.15674000 & -0.94683897 & 1.14970000 & -0.79198649 \\
0.90 & -0.21670000 & 0.15650000 & -1.02152482 & 1.04561080 & -0.71586312 \\
0.80 & -0.20540000 & 0.17274000 & -1.19568249 & 0.85004562 & -0.57640893 \\
0.70 & -0.19740000 & 0.20078000 & -1.40425931 & 0.63290021 & -0.42620995 \\
0.60 & -0.18981000 & 0.21898000 & -1.64684945 & 0.28035791 & -0.17610118 \\
0.50 & -0.18176000 & 0.22200000 & -1.94918274 & -0.33158000 & 0.22466705 \\
0.40 & -0.18230000 & 0.31655000 & -2.45772558 & -0.57085000 & 0.37974756 \\
0.30 & -0.19170000 & 0.49447000 & -3.33712898 & -0.98000000 & 0.65679442 \\
0.20 & -0.20987000 & 0.81123000 & -5.18847693 & -1.84100000 & 1.25314140 \\
0.10 & -0.23832000 & 1.52926000 & -11.49251591 & -4.62910000 & 3.22719187 \\
0.05 & -0.26170350 & 2.46670000 & -26.30509253 & -10.65015000 & 7.56893373 \\
\hline
\end{tabular}

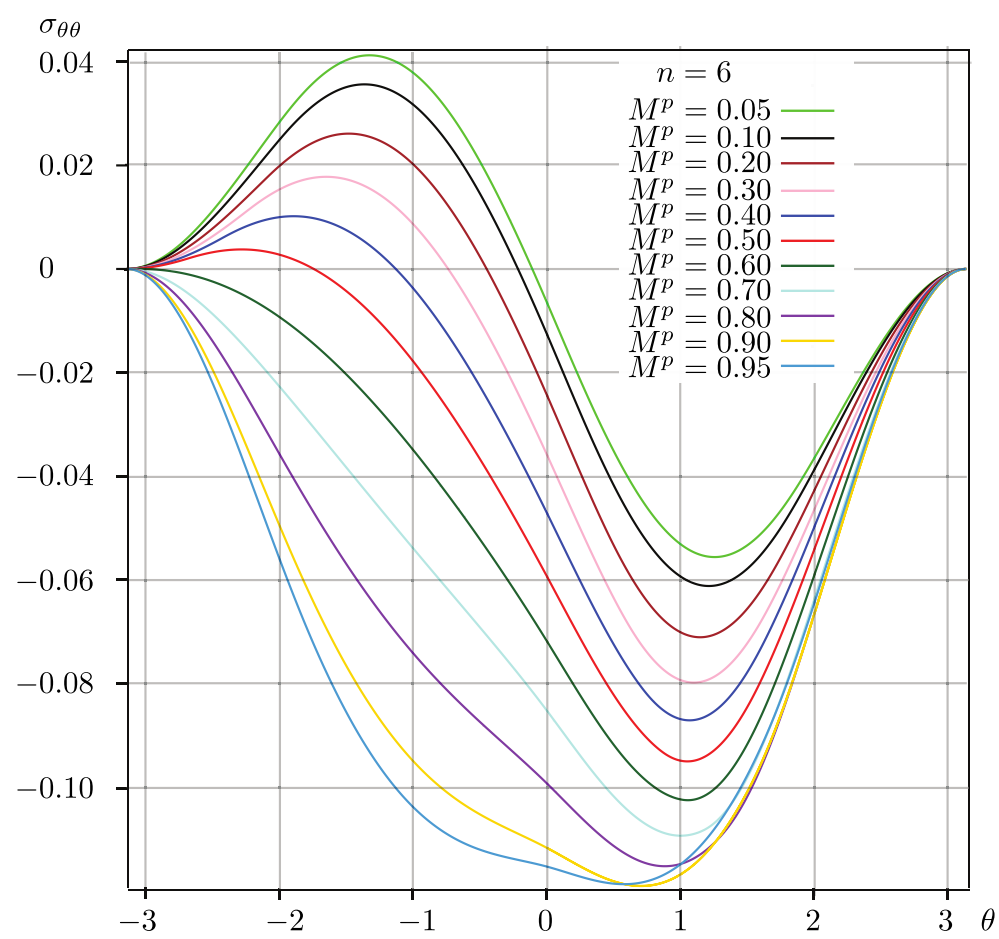

Рис. 1. (online в цвете) Угловые распределения компоненты тензора напряжений $\sigma_{\theta \theta}$ для $n=6$ [Figure 1. (color online) Angular distribution of the circumferential stress in the vicinity of the crack tip; $n=6]$ 


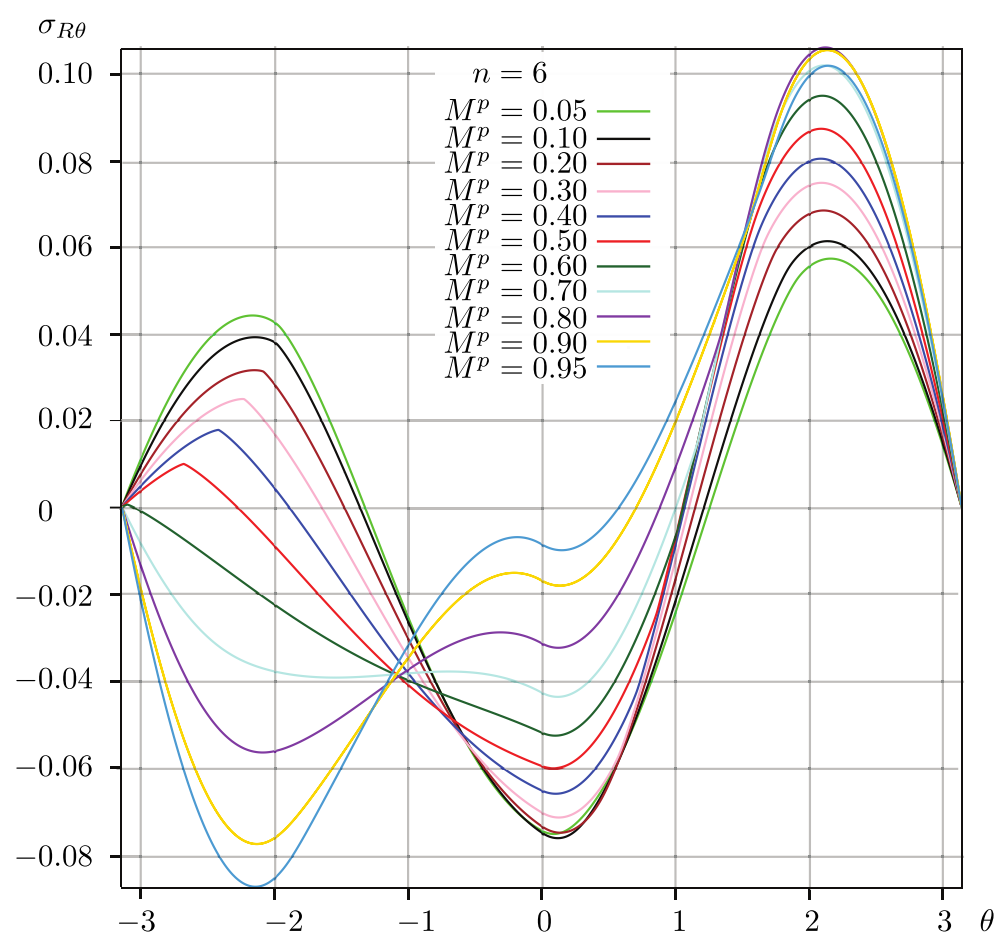

Рис. 2. (online в цвете) Угловые распределения компоненты тензора напряжений $\sigma_{R \theta}$ для $n=6$ [Figure 2. (color online) Angular distribution of the tangential stress in the vicinity of the crack tip; $n=6$ ]

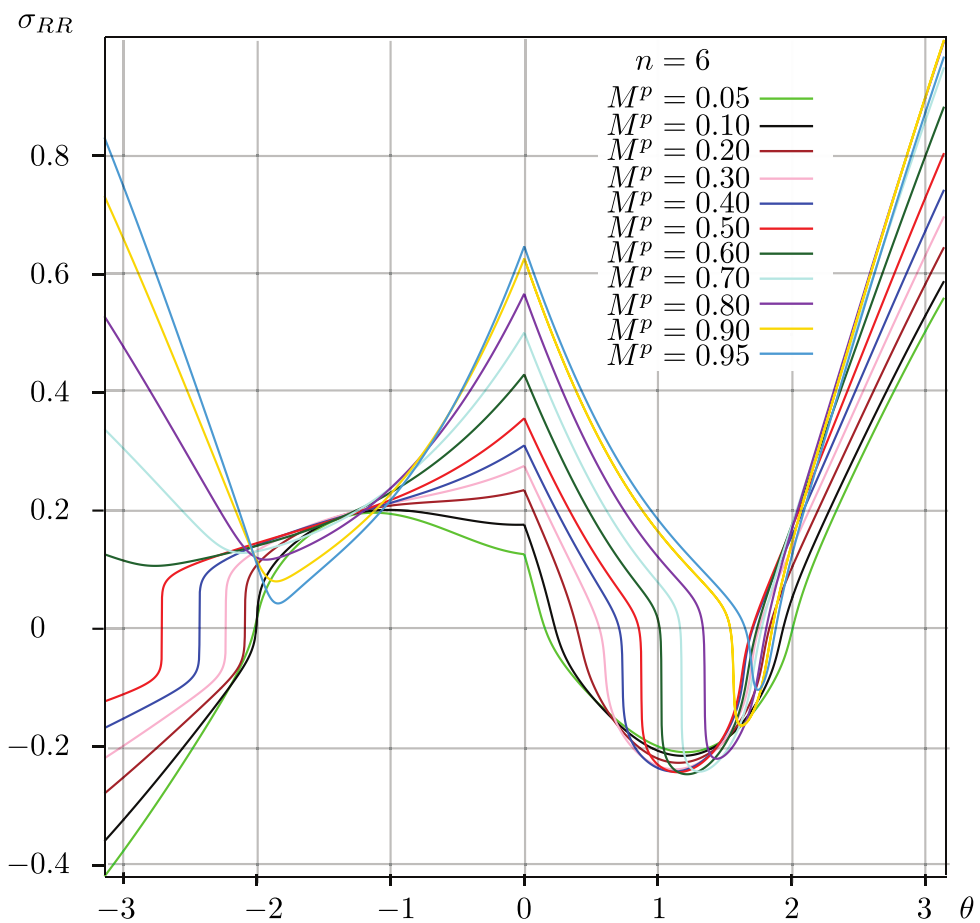

Рис. 3. (online в цвете) Угловые распределения компоненты тензора напряжений $\sigma_{R R}$ для $n=6$ [Figure 3. (color online) Angular distribution of the radial stress in the vicinity of the crack tip; $n=6$ ] 


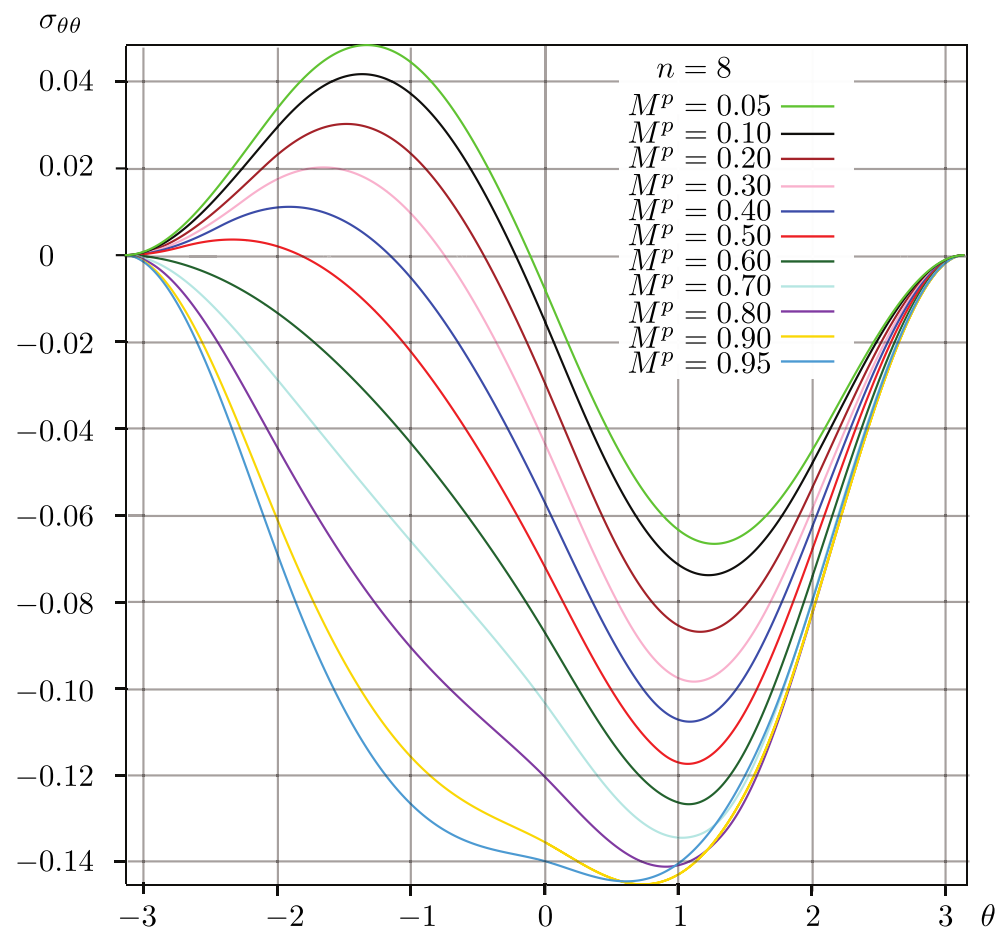

Рис. 4. (online в цвете) Угловые распределения компоненты тензора напряжений $\sigma_{\theta \theta}$ для $n=8$ [Figure 4. (color online) Angular distribution of the circumferential stress in the vicinity of the crack tip; $n=8$ ]

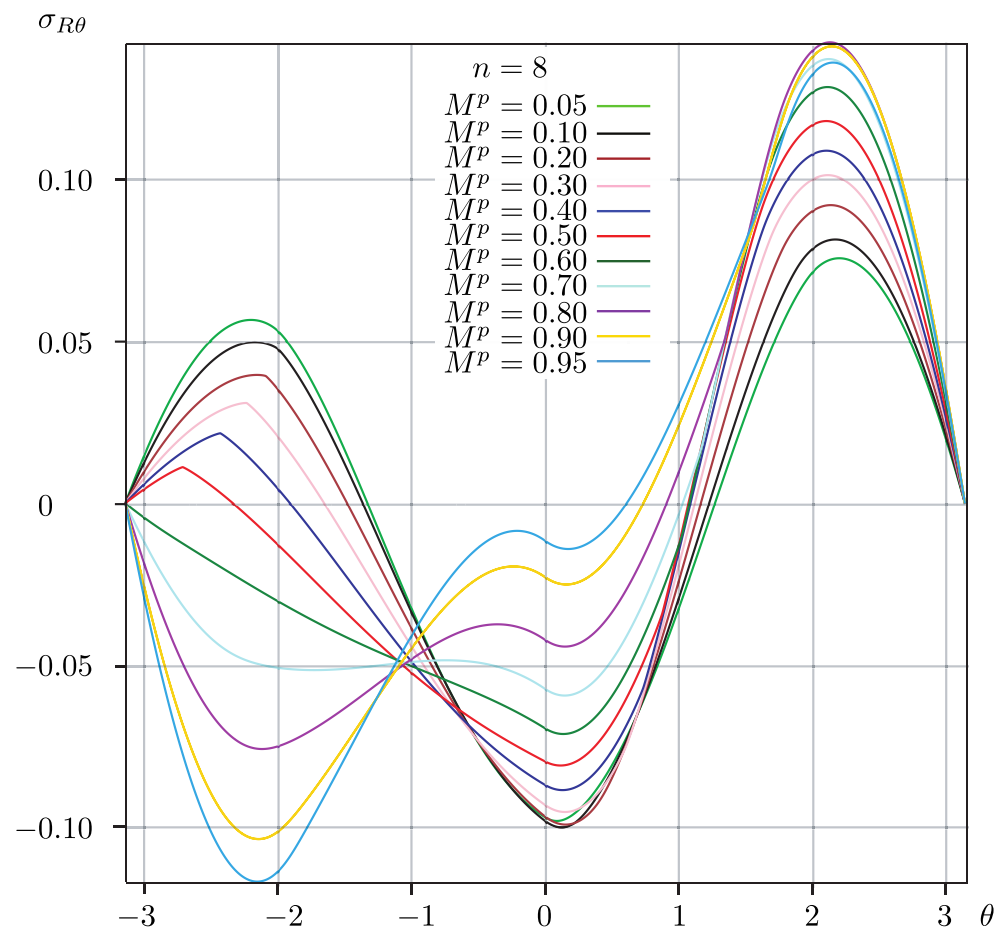

Рис. 5. (online в цвете) Угловые распределения компоненты тензора напряжений $\sigma_{R \theta}$ для $n=8$ [Figure 5. (color online) Angular distribution of the tangential stress in the vicinity of the crack tip; $n=8$ ] 


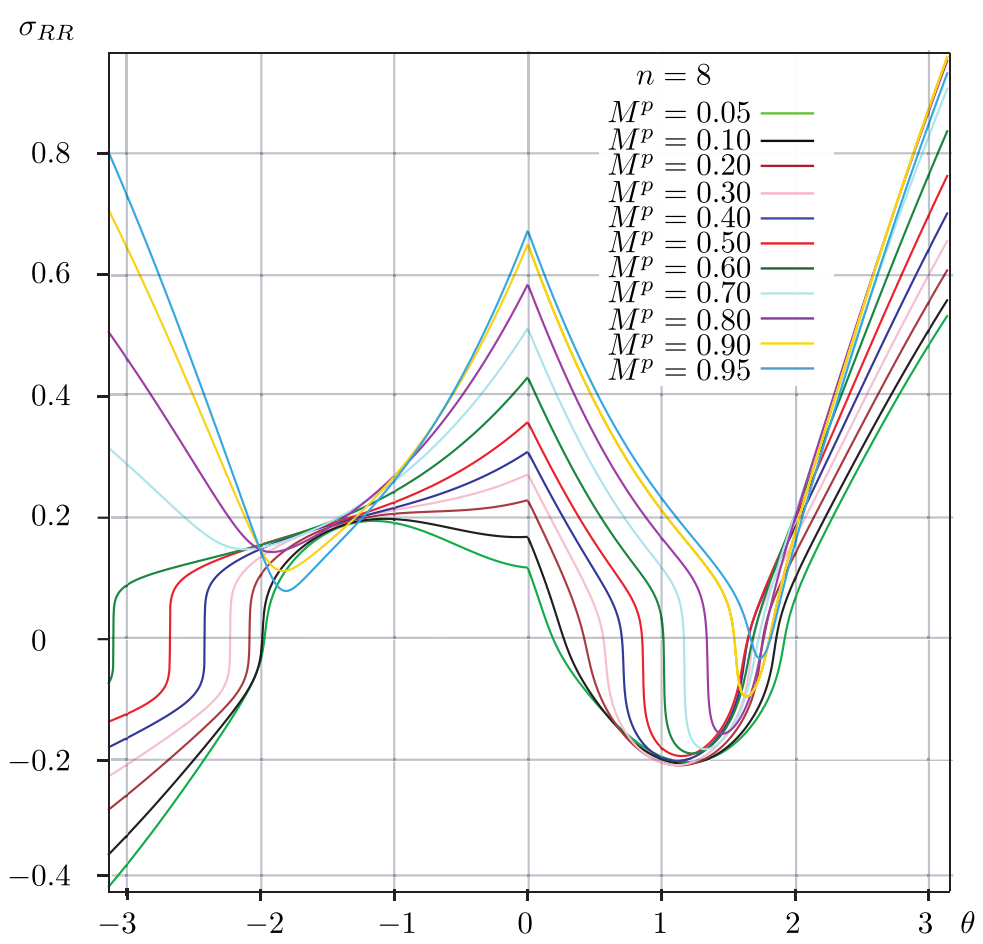

Рис. 6. (online в цвете) Угловые распределения компоненты тензора напряжений $\sigma_{R R}$ для $n=8$ [Figure 6. (color online) Angular distribution of the radial stress in the vicinity of the crack tip; $n=8$ ]

переменная

$$
R=r(A t)^{-(n+1) / m} B I_{n} / C^{*}
$$

и система уравнений задачи допускает автомодельное представление решения:

$$
\sigma_{i j}(r, \theta, t)=(A t)^{-1 / m} \hat{\sigma_{i j}}(R, \theta), \quad \psi(r, \theta, t)=\hat{\psi}(R, \theta) .
$$

Следует отметить, что граничное условие в бесконечно удаленной точке может быть сформулировано в более общей по сравнению с (11) форме

$$
\sigma_{i j}(r \rightarrow \infty, \theta, t) \rightarrow \tilde{C} r^{s} \overline{\sigma_{i j}}(\theta, n)
$$

где показатель степени $s$ подлежит определению в ходе решения задачи; $\tilde{C}-$ амплитуда поля напряжений на бесконечности, определяемая геометрией реального образца и системой приложенных нагрузок. Для степенных определяющих соотношений (9), кинетического уравнения (10) и более общих граничных условий (12) существует автомодельная переменная

$$
R=r\left(A t \tilde{C}^{m}\right)^{1 / s m} .
$$

После введения автомодельной переменной уравнения равновесия (3), определяющие уравнения (9), условия совместности (4) сохраняют свою форму, 
а кинетическое уравнение накопления повреждений принимает вид

$$
R \hat{\psi}, R=-s m\left(\hat{\sigma}_{e} / \hat{\psi}\right)^{m} .
$$

Асимптотическое решение задачи вне области диспергированного материала, формирующееся у вершины трещины (на больших расстояниях от вершины трещины $R \rightarrow \infty)$, разыскивается в форме

$$
\begin{aligned}
& \chi(R, \theta)=R^{\lambda+1} f^{(0)}(\theta)+R^{\lambda_{1}+1} f^{(1)}(\theta)+R^{\lambda_{2}+1} f^{(2)}(\theta)+o\left(R^{\lambda_{2}}\right), \\
& \psi(R, \theta)=1-R^{\gamma} g^{(0)}(\theta)-R^{\gamma_{1}} g^{(1)}(\theta)-R^{\gamma_{2}} g^{(2)}(\theta)+o\left(R^{\gamma_{2}}\right),
\end{aligned}
$$

где $\chi(R, \theta)$ - трехчленное асимптотическое разложение функции напряжения Эри; $\lambda, \lambda_{k}, \gamma, \gamma_{k}, f_{(k)}(\theta), g_{k}(\theta)$ - неизвестные собственные значения и собственные функции соответственно, подлежащие определению. В силу (13) асимптотическое представление компонент тензора напряжений вне области деспригированного материала имеет вид

$$
\begin{aligned}
& \sigma_{R R}(R, \theta)=R^{s}\left[(\lambda+1) f^{(0)}+\left(f^{(0)}\right)^{\prime \prime}\right]+R^{s_{1}}\left[(\lambda+1) f^{(1)}+\left(f^{(1)}\right)^{\prime \prime}\right]+ \\
&+R^{s_{2}}\left[(\lambda+1) f^{(2)}+\left(f^{(2)}\right)^{\prime \prime}\right]+\ldots, \\
& \sigma_{\theta \theta}(R, \theta)=R^{s} \lambda(\lambda+1) f^{(0)}+R^{s_{1}} \lambda(\lambda+1) f^{(1)}+R^{s_{2}} \lambda(\lambda+1) f^{(2)}+\ldots, \\
& \sigma_{R \theta}(R, \theta)=-R^{s}(\lambda+1)\left(f^{(0)}\right)^{\prime}-R^{s_{1}}(\lambda+1)\left(f^{(1)}\right)^{\prime}-R^{s_{2}}(\lambda+1)\left(f^{(2)}\right)^{\prime}+\ldots,
\end{aligned}
$$

где $s=\lambda-1, s_{k}=\lambda_{k}-1$.

Асимптотический анализ уравнений задачи позволяет установить, что справедливы равенства $\lambda_{k}-\lambda=k\left(\lambda_{1}-\lambda\right)$ для всех $k$ и асимптотическое представление интенсивности касательных напряжений принимает форму

$$
\sigma_{e}(R, \theta)=R^{\lambda-1} \sigma_{e}^{(0)}(\theta)\left[1+R^{\lambda_{1}-\lambda} \sigma_{e}^{(1)}(\theta)+R^{2\left(\lambda_{1}-\lambda\right)} \sigma_{e}^{(2)}(\theta)+\ldots\right],
$$

где

$$
\begin{aligned}
\sigma_{e}^{(0)}= & \sqrt{\left((1-\lambda) f_{0}+\left(f_{0}^{\prime \prime}\right)^{2}\right)+(\lambda+1)^{2} f_{0}^{2}-\left((\lambda+1) f_{0} f_{0}^{\prime \prime}\right)(\lambda+1) \lambda f_{0}+3 \lambda^{2}\left(f_{0}^{\prime}\right)^{2}}, \\
\sigma_{e}^{(1)}= & f_{e}^{(1)} /\left(\sigma_{e}^{(0)}\right)^{2}, \\
f_{e}^{(1)}= & {\left[\left((\lambda+1) f_{0}+f_{0}^{\prime \prime}\right)\left(\left(\lambda_{1}+1\right) f_{1}+f_{1}^{\prime \prime}\right)+\lambda(\lambda+1) \lambda_{1}\left(\lambda_{1+1}\right) f_{0} f_{1}-\right.} \\
& \left.-\left((\lambda+1) f_{0}+f_{0}^{\prime \prime} \lambda_{1}\left(\lambda_{1}+1\right) f_{1}+\left(\lambda_{1}+1\right) f_{1}+f_{1}^{\prime \prime}\right) \lambda(\lambda+1) f_{0}\right]+3 \lambda \lambda_{1} f_{0}^{\prime} f_{1}^{\prime} .
\end{aligned}
$$

Кинетическое уравнение накопления повреждений и гипотеза о том, что порядки малости слагаемых, последовательно появляющихся в этом уравнении, в правой части одинаковы, позволяют связать показатели степеней в асимптотических разложениях функции напряжения Эри и параметра сплошности (13):

$$
\gamma=(\lambda-1) m, \quad \gamma_{k}=(\lambda-1) m+k\left(\lambda_{1}-1\right) .
$$

Анализ асимптотических разложений компонент тензора скоростей деформаций ползучести дает возможность установить, что

$$
\lambda_{1}-\lambda=\gamma=(\lambda-1) m .
$$


Откуда легко найти, что $\gamma_{k}=k(\lambda-1) m$, и получить следующие асимптотические представления компонент тензора скоростей деформаций ползучести вне области полностью поврежденного (диспергированного) материала:

$$
\begin{aligned}
& \dot{\varepsilon}_{R R}(R, \theta)=R^{(\lambda-1) n} \varepsilon_{R R}^{(0)}(\theta)+R^{(\lambda-1)(n+m)} \varepsilon_{R R}^{(1)}(\theta)+R^{(\lambda-1)(n+2 m)} \varepsilon_{R R}^{(2)}(\theta)+\ldots, \\
& \dot{\varepsilon}_{R \theta}(R, \theta)=R^{(\lambda-1) n} \varepsilon_{R \theta}^{(0)}(\theta)+R^{(\lambda-1)(n+m)} \varepsilon_{R \theta}^{(1)}(\theta)+R^{(\lambda-1)(n+2 m)} \varepsilon_{R \theta}^{(2)}(\theta)+\ldots \\
& \dot{\varepsilon}_{\theta \theta}(R, \theta)=R^{(\lambda-1) n} \varepsilon_{\theta \theta}^{(0)}(\theta)+R^{(\lambda-1)(n+m)} \varepsilon_{\theta \theta}^{(1)}(\theta)+R^{(\lambda-1)(n+2 m)} \varepsilon_{\theta \theta}^{(2)}(\theta)+\ldots
\end{aligned}
$$

где угловые распределения главного члена асимптотического разложения компонент тензора скоростей деформаций определяются формулами

$$
\begin{aligned}
& \varepsilon_{R R}^{(0)}(\theta)=\left(\sigma_{e}^{(0)}\right)^{n-1}\left[(1+\lambda)(2-\lambda) f_{0}+2 f_{0}^{\prime \prime}\right] \\
& \varepsilon_{\theta \theta}^{(0)}(\theta)=-2\left(\sigma_{e}^{(0)}\right)^{n-1}\left[(1+\lambda)(2 \lambda-1) f_{0}-\left(f_{0}\right)^{\prime \prime}\right], \\
& \varepsilon_{R \theta}^{(0)}(\theta)=-3\left(\sigma_{e}^{(0)}\right)^{n-1} \lambda f_{0}^{\prime}
\end{aligned}
$$

коэффициенты первого слагаемого в асимптотическом разложении компонент тензора скоростей деформаций -

$$
\begin{aligned}
& \varepsilon_{R R}^{(1)}(\theta)=\left(\sigma_{e}^{(0)}\right)^{n-1}\left[2\left[\left(1+\lambda_{1}\right) f_{1}+f_{1}^{\prime \prime}\right]-\lambda_{1}\left(1+\lambda_{1}\right) f_{1}+\right. \\
& \left.+\left[2(1+\lambda) f_{0}+f_{0}^{\prime \prime}-\lambda(1+\lambda) f_{0}\right]\left[(n+1) \sigma_{e}^{(1)}+n g_{0}\right] \sigma_{e}^{(0)}\right], \\
& \varepsilon_{\theta \theta}^{(1)}(\theta)=\left(\sigma_{e}^{(0)}\right)^{n-1}\left[2 \lambda_{1}\left(1+\lambda_{1}\right) f_{1}-f_{1}^{\prime \prime}-\left(1+\lambda_{1}\right) f_{1}+\right. \\
& \left.+\left[2 \lambda(1+\lambda) f_{0}-f_{0}^{\prime \prime}-(1+\lambda) f_{0}\right]\left[(n+1) \sigma_{e}^{(1)}+n g_{0}\right] \sigma_{e}^{(0)}\right], \\
& \varepsilon_{R \theta}^{(1)}(\theta)=-3\left(\sigma_{e}^{(0)}\right)^{n-1}\left[-\lambda_{1} f_{1}^{\prime}-\lambda f_{0}^{\prime}\left[(n+1) \sigma_{e}^{(1)}+n g_{0}\right] \sigma_{e}^{(0)}\right] \text {. }
\end{aligned}
$$

Из условия совместности деформаций (4) можно получить систему обыкновенных дифференциальных уравнений для определения угловых распределений компонент тензора напряжений. Для нахождения функции $f_{0}(\theta)$ получается нелинейное обыкновенное дифференциальное уравнение, совпадающее с рассматриваемым ранее уравнением (5):

$$
2[(\lambda-1) n+1] \frac{d \varepsilon_{R \theta}^{(0)}}{d \theta}=\frac{d^{2} \varepsilon_{R R}^{(0)}}{d \theta^{2}}-(\lambda-1) n[(\lambda-1) n+2] \varepsilon_{R R}^{(0)} .
$$

Относительно функций $f_{k}(\theta)(k \geqslant 1)$ из условия совместности (4) следуют линейные обыкновенные дифференциальные уравнения

$$
\begin{aligned}
2[(\lambda-1)(n+k m)+1] \frac{d \varepsilon_{R \theta}^{(k)}}{d \theta} & =\frac{d^{2} \varepsilon_{R R}^{(k)}}{d \theta^{2}}- \\
- & (\lambda-1)(n+k m)[(\lambda-1)(n+k m)+2] \varepsilon_{R R}^{(k)} .
\end{aligned}
$$

Решение системы уравнений (14), (15) должно удовлетворять граничным условиям $f_{k}(\theta= \pm \pi)=0$ и $f_{k}^{\prime}(\theta= \pm \pi)=0$. Численное решение системы уравнений $(14),(15)$ на отрезке $[-\pi, \pi]$ разыскивалось с помощью описанного выше алгоритма в пакете Mathematica V. 5.1. 
В случае смешанного деформирования соображения симметрии и антисимметрии использованы быть не могут, поэтому необходимо искать решение уравнения (14) на отрезке $[-\pi, \pi]$. В условиях смешанного нагружения при численном решении уравнения (15) отрезок интегрирования $[-\pi, \pi]$ можно разбить на два отрезка: $[-\pi, 0]$ и $[0, \pi]$. Сначала уравнение $(14)$ интегрируется на отрезке $[0, \pi]$, и двухточечная краевая задача сводится к задаче Коши с начальными условиями.

Найдено новое промежуточно-асимптотическое поведение напряжений в задаче о трещине нормального отрыва в среде с поврежденностью в связанной постановке. Конфигурации областей полностью поврежденного материала представлены на рис. 7-10. Здесь кривая 1 - контур области полностью поврежденного материала, полученный с помощью двучленного асимптотического разложения параметра сплошности $\psi(R, \theta)=1-R^{\gamma} g^{(0)}(\theta)$; кривая 2 - контур области полностью поврежденного материала, полученный с помощью трехчленного асимптотического разложения параметра сплошности $\psi(R, \theta)=1-R^{\gamma} g^{(0)}(\theta)-R^{\gamma_{1}} g^{(1)}(\theta)$.

Так же была построена геометрия области полностью поврежденного материала для уточненного решения HRR, приведенного в работе [33] (рис. 11). Из рисунка видно, что конфигурации области полностью поврежденного материала, полученные с помощью двучленного и трехчленного асимптотического разложения, сильно отличаются друг от друга, что противоречит предположению, что каждое следующее слагаемое асимптотического разложения параметра сплошности должно быть лишь малым дополнением к предыду-

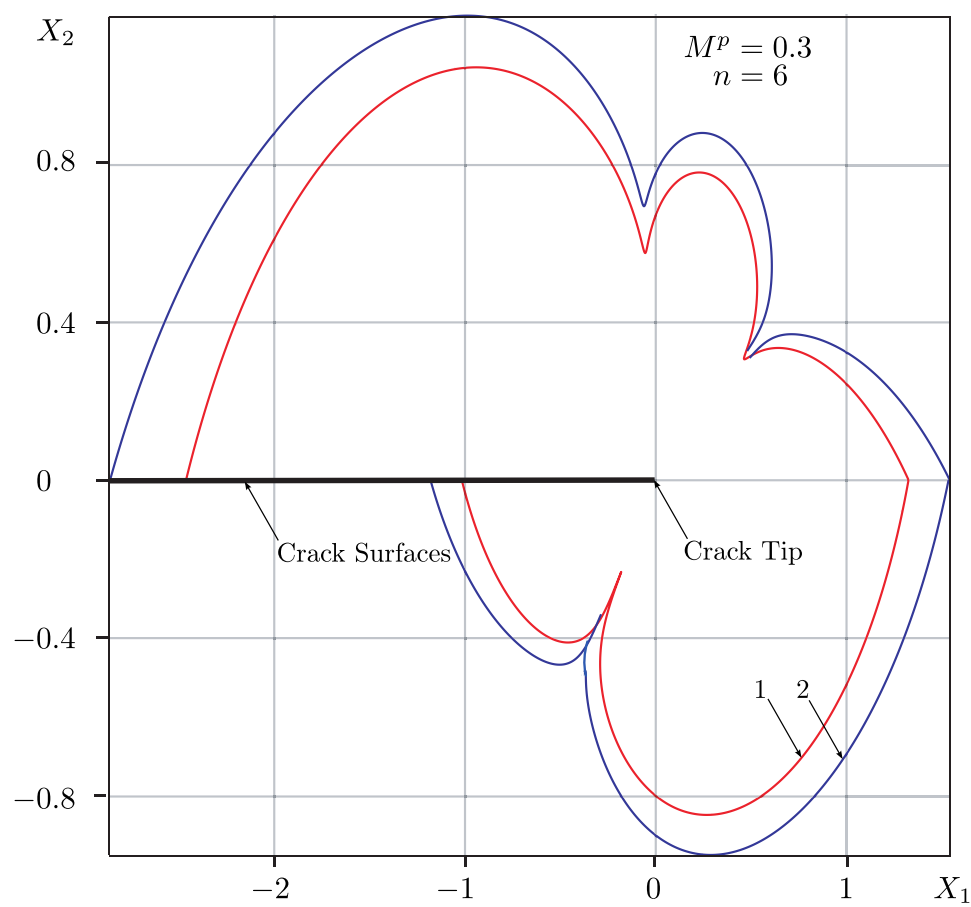

Рис. 7. (online в цвете) Геометрия области полностью поврежденного материала для $M^{p}=0.3, n=6$

[Figure 7. (color online) Geometry of the completely damage zone for $M^{p}=0.3, n=6$ ] 


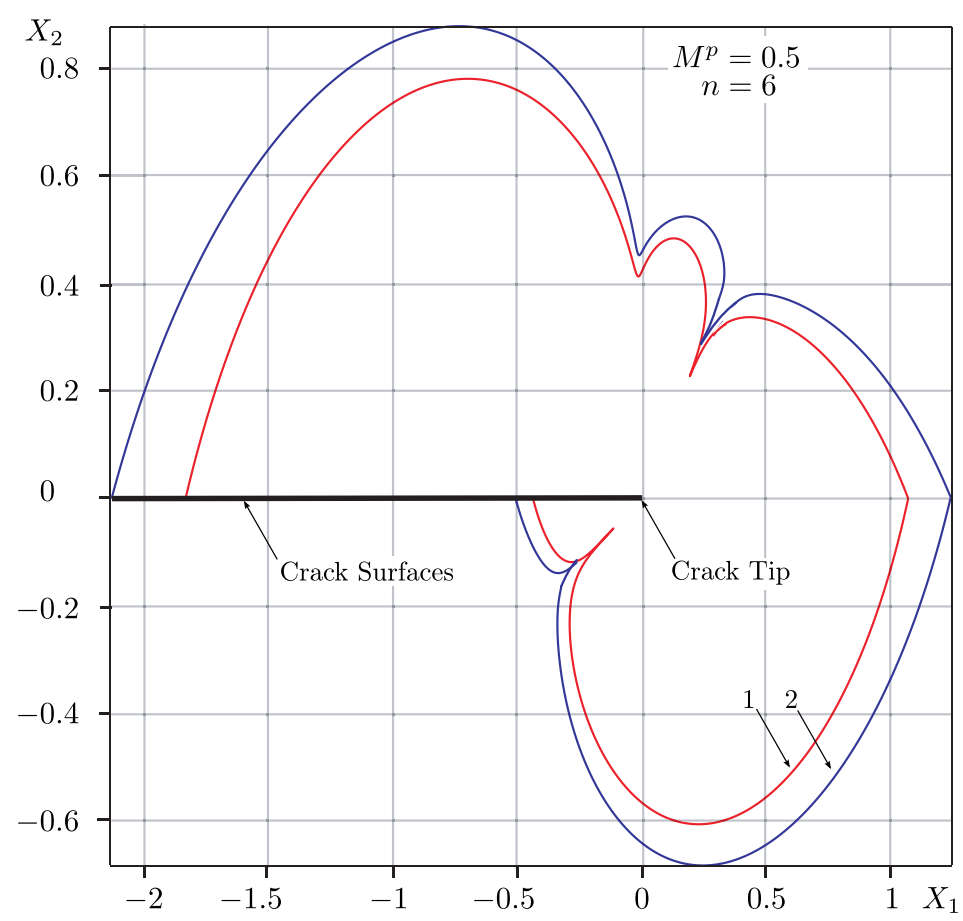

Рис. 8. (online в цвете) Геометрия области полностью поврежденного материала для $M^{p}=0.5, n=6$

[Figure 8. (color online) Geometry of the completely damage zone for $M^{p}=0.5, n=6$ ]

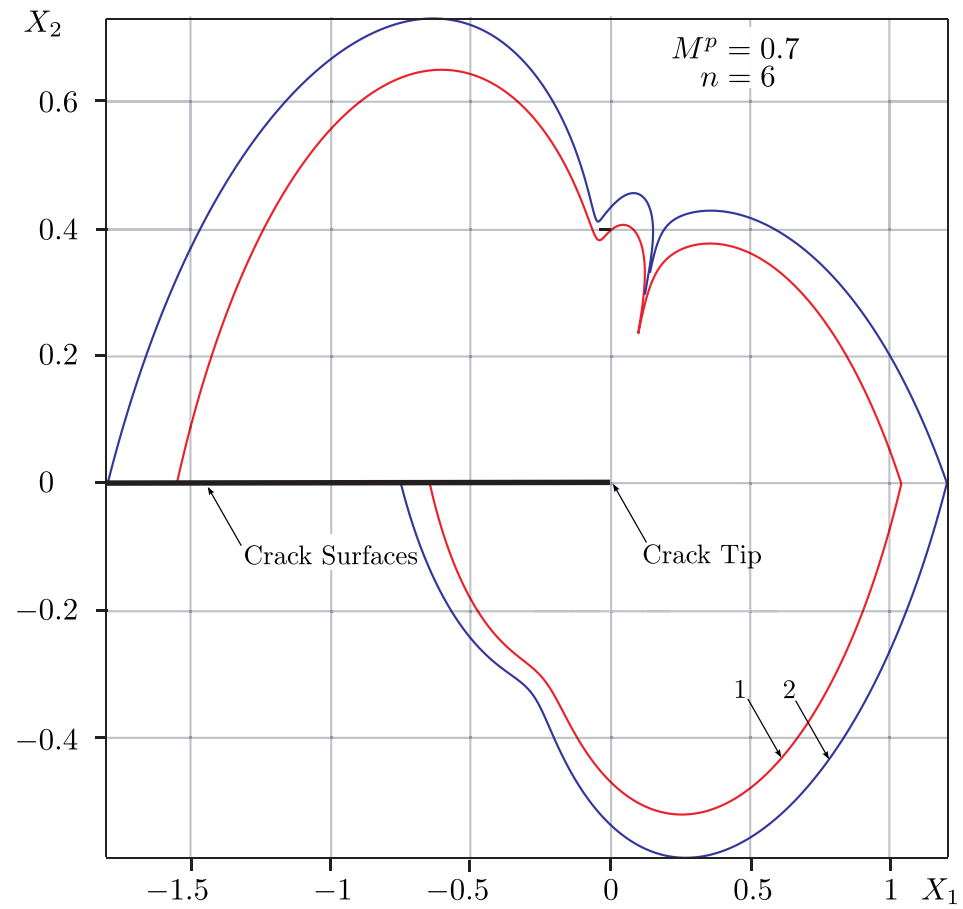

Pис. 9. (online в цвете) Геометрия области полностью поврежденного материала для $M^{p}=0.7, n=6$

[Figure 9. (color online) Geometry of the completely damage zone for $M^{p}=0.7, n=6$ ] 


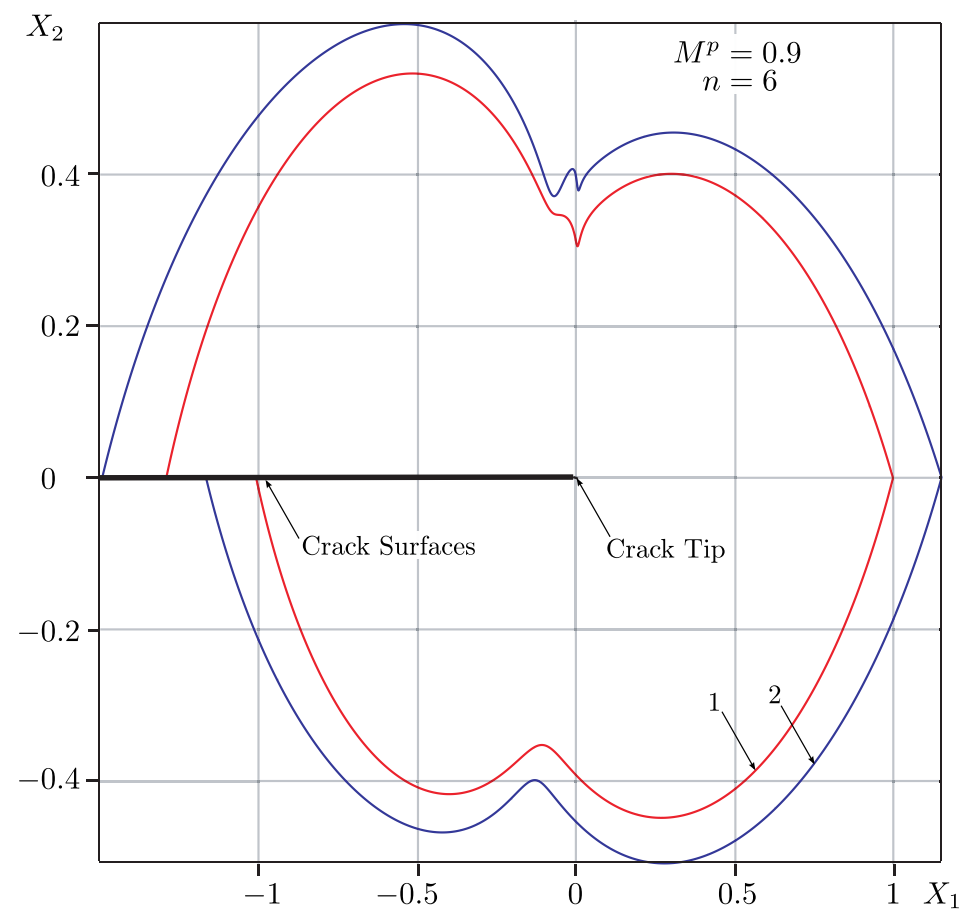

Рис. 10. (online в цвете) Геометрия области полностью поврежденного материала для $M^{p}=0.9, n=6$

[Figure 10. (color online) Geometry of the completely damage zone for $M^{p}=0.9, n=6$ ]

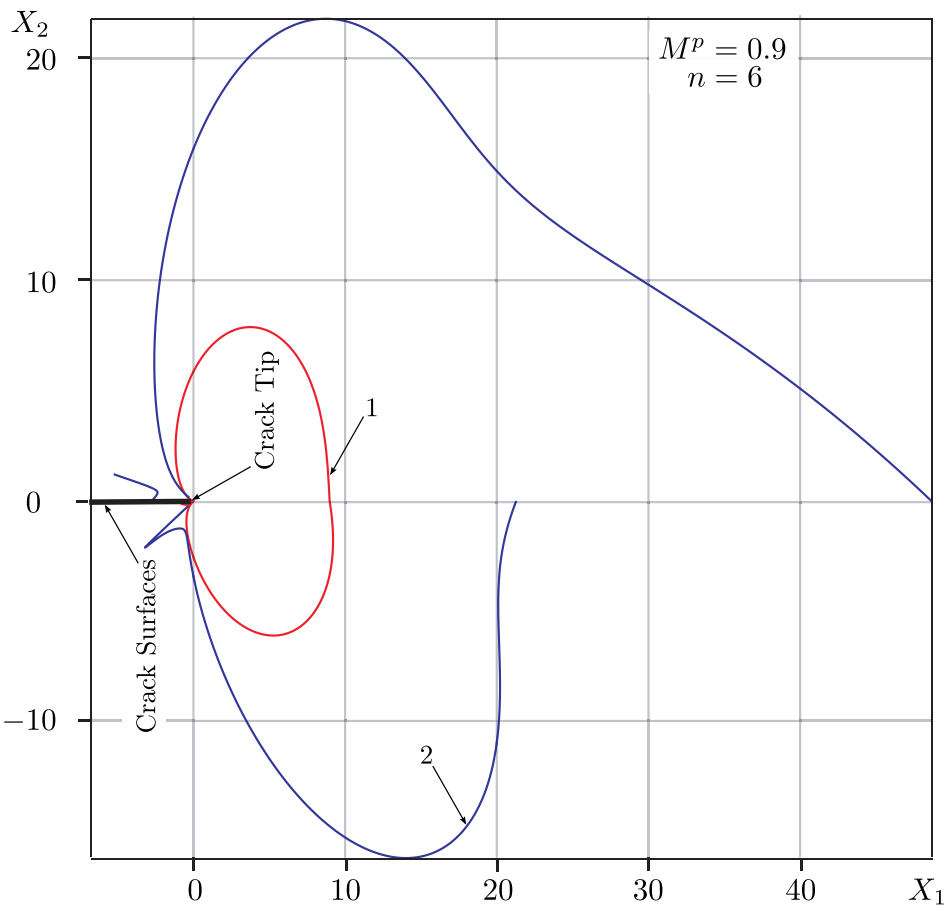

Рис. 11. (online в цвете) Геометрия области полностью поврежденного материала для $M^{p}=0.9, n=6$ для асимптотики Хатчинсона-Райса-Розенгрена [33]

[Figure 11. (color online) Geometry of the completely damage zone for $M^{p}=0.9, n=6$; Hutchinson-Rice-Rosengren asymptotic solution [33] ] 
щему. Таким образом в данной работе показано, что новая промежуточная асимтотика позволяет более точно построить конфигурации областей диспергированного материала.

Выводы и обсуждение результатов. В настоящей работе рассмотрена задача отыскания распределения напряжений и сплошности у вершины трещины в условиях плоского напряженного состояния. Для решения задачи используется метод разложения по собственным функциям. Показано, что задача определения напряженно-деформированного состояния редуцируется к нелинейной задаче на собственные значения, численное решение которой получено. В статье развит численный метод нахождения всего спектра собственных значений задачи. Представленный метод применен к анализу полей напряжений и сплошности у вершины трещины в среде с поврежденностью. Принята гипотеза о существовании области полностью поврежденного материала и с помощью автомодельного представления решения найдена ее геометрия.

Благодарности. Работа выполнена при финансовой поддержке Российского фонда фундаментальных исследований (проект № 13-01-97009-а-Поволжье).

\section{ORCID}

Лариса Валентиновна Степанова: http://orcid.org/0000-0002-6693-3132

\section{БИБЛИОГРАФИЧЕСКИЙ СПИСОК}

1. Степанова Л. В., Яковлева Е. М. О смешанном нагружении элементов конструкций с дефектами / Четвертая международная конферениия «Математическая физика и ее приложения»: материалы конф.; ред. чл.-корр. РАН И. В. Волович; д.ф.-м.н., проф. В. П. Радченко. Самара: СамГТУ, 2014. С. 336-337.

2. Bui H. D. Fracture Mechanics. Inverse Problems and Solutions/ Solid Mechanics and Its Applications. T.139. Netherlands: Springer, 2006. xxviii +382 c.. doi: 10.1007/ 978-1-4020-4837-1

3. Li J., Recho N. Méthodes asymptotiques en mécanique de la rupture. Paris: Hermes Science Publications, 2002. 262 pp.

4. Handbook of Damage Mechanics: Nano to Macro Scale for Materials and Structures / ed. G. Z. Voyiadjis. New York: Springer, 2015. xxviii+1591 pp.. doi: 10.1007/ 978-1-4614-5589-9.

5. François D., Pineau A., Zaoui A. Mechanical Behaviour of Materials. Vol. 1: Microand Macroscopic Constitutive Behaviour / Solid Mechanics and Its Applications. vol. 180. Netherlands: Springer, 2013. xvii+662 pp.. doi : 10.1007/978-94-007-2546-1.

6. Sih G. C. Crack tip mechanics based on progressive damage of arrow: Hierarchy of singularities and multiscale segment// Theoretical and Applied Fracture Mechanics, 2009. vol. 51, no. 1. pp. 11-32. doi: 10.1016/j.tafmec.2009.01.007.

7. Sih G. C., Tang X. S. Simultaneity of multiscaling for macro-meso-micro damage model represented by strong singularities // Theoretical and Applied Fracture Mechanics, 2004. vol. 42, no. 3. pp. 199-225. doi : 10.1016/j.tafmec.2004.09.001.

8. Sih G. C., Tang X. S. Weak and strong singularities reflecting multiscale damage: microboundary conditions for free-free, fixed-fixed and free-fixed constraints // Theoretical and Applied Fracture Mechanics, 2005. vol.43, no.1. pp. 5-62. doi:10.1016/j.tafmec. 2004. 12.002 .

9. Аргатов И. И. Введение в асимптотическое моделирование в механике. СПб.: Политехника, 2004. 302 с.

10. Степанова Л. В. Математические методъ механики разрушения. Самара: Самарский университет, 2006. 232 с.

11. Williams M. L. Stress singularities resulting from various boundary conditions in angular corners of plates in extensions // Journal of Applied Mechanics, 1952. vol. 19. pp. 526-534. 
12. Williams M. L. On the stress distribution at the base of a stationary crack // Journal of Applied Mechanics, 1957. vol.24. pp. 109-114.

13. Beliakova T. A. The eigenspectrum approach and T-stress at the mixed-mode crack tip for a stress-state dependent material // Procedia Materials Sience, 2014. vol. 3. pp. 147-152. doi: $10.1016 / j$.mspro.2014.06.027.

14. Hutchinson J. W. Singular behaviour at the end of a tensile crack in a hardening material // Journal of the Mechanics and Physics of Solids, 1968. vol.16, no.1. pp. 13-31. doi: 10 . 1016/0022-5096(68) 90014-8.

15. Hutchinson J. W. Plastic stress and strain fields at a crack tip // Journal of the Mechanics and Physics of Solids, 1968. vol.16, no.5. pp. 337-347. doi:10.1016/0022-5096(68) 90021-5.

16. Rice J. R., Rosengren G. F. Plane strain deformation near a crack tip in a power-law harderning material // Journal of the Mechanics and Physics of Solids, 1968. vol. 16, no. 1. pp. 1-12. doi: 10.1016/0022-5096(68)90013-6.

17. Zehnder A. Fracture Mechanics / Lecture Notes in Applied and Computational Mechanics. vol. 62. Netherlands: Springer, 2012. xiv+226 pp.. doi : 10.1007/978-94-007-2595-9

18. Multiscale Fatigue Crack Initiation and Propagation of Engineering Materials: Structural Integrity and Microstructural Worthiness/ Solid Mechanics and its Applications. vol. 152 / eds. G. C. Sih. Netherlands: Springer, 2008. xiii+380 pp.. doi:10.1007/ 978-1-4020-8520-8.

19. Адылина Е. М., Игонин С. А., Степанова Л. В. О нелинейной задаче на собственные значения, следующей из анализа напряжений у вершины усталостной трещины // Вестн. СамГУ. Естественнонаучн. сер., 2012. № 3/1(94). С. 83-102.

20. Failure and Damage Analises of Advanced Materials/ CISM International Centre for Mechanical Sciences. vol. 560 / eds. H. Altenbach, T. Sadowski. Vienna: Springer, 2015. xii+282 pp.. doi : 10.1007/978-3-7091-1835-1.

21. Hello G., Taha M. B., Roelandt J. M. Analytical determination of coefficients in crack-tip stress expansions for a finite crack in an infinite plane medium // International Journal of Solids and Structures, 2012. vol.49, no. 3-4. pp. 556-566. doi: 10.1016/j.ijsolstr. 2011. 10.024.

22. Shih C. F. Elastic-plastic analysis of combined mode crack problems: PhD Thesis. Harvard University, 1973.

23. Shih C. F. Small-scale yielding analysis of mixed mode plane-strain crack problems // National Symposium on Fracture Mechanics, 1974. vol. STP560. pp. 187-210. doi: 10.1520/ stp33141s.

24. Степанова Л. В., Адылина Е. М. Асимптотические методы нелинейной механики разрушения: результаты, современное состояние и перспективы // Вестн. Сам. гос. техн. ун-та. Сер. Физ.-мат. науки, 2013. №2(31). С. 156-168. doi: 10.14498/vsgtu1156.

25. Шлянников В. Н., Туманов А. В. Упругие параметры смешанных форм деформирования полуэллиптической трещины при двухосном нагружении // Изв. Capam. yн-та. Нов. сер. Сер. Математика. Механика. Информатика, 2010. Т. 10, № 2. С. 73-80.

26. Шлянников В. Н., Кислова С. Ю. Параметры смешанных форм деформирования для трещины в виде математического разреза // Изв. Сарат. ун-та. Нов. сер. Сер. Математика. Механика. Информатика, 2009. Т. 9, №1. С. 77-84.

27. Fatigue, Failure, and Damage Evolution: Proceedings of the 2014 Annual Conference on Experimental and Applied Mechanics. vol. 5 / eds. J. Carroll, S. Daly. New York: Springer, 2015. viii+252 pp.. doi: 10.1007/978-3-319-06977-7

28. Rahman S., Mohammad E. Effects of mixed-mode overloading on the mixed-mode I+II fatigue crack growth// Archive of Applied Mechanics, 2013. vol.83, no. 7. pp. 987-1000. doi: 10.1007/s00419-013-0731-z.

29. Степанова Л. В. О собственных значениях в задаче о трещине антиплоского сдвига в материале со степенными определяющими уравнениями // ПМТФ, 2008. Т. 49, № 1 . C. $173-180$. 
30. Степанова Л. В. Анализ собственных значений в задаче о трещине в материале со степенным определяющим законом // ЖК. вычисл. матем. и матем. физ., 2009. Т. 49, № 8. С. 1399-1415.

31. Степанова Л. В. Уточненный расчет напряженно-деформированного состояния у вершины трещины в условиях циклического нагружения в среде с поврежденностью// Вестн. СамГУ. Естественнонаучн. сер., 2011. №2(83). С. 105-115.

32. Rice J. R. Mathematical analysis in mechanics of fracture / Fracture: An Advanced Treatise. vol. 2, Mathematical Fundamentals; ed. H. Liebowitz. New York: Academic Press, 1968. pp. 191-311, http://esag.harvard.edu/rice/018_Rice_MathAnalMechFract_68.pdf.

33. Степанова Л. В., Яковлева Е. М. Смешанное деформирование пластины с трещиной в условиях плоского напряженного состояния// Вестник Пермского начионалъного исследовательского политехнического университета. Механика, 2014. № 3. С. 129-162. doi : 10.15593/perm.mech/2014.3.08.

34. Качанов Л. М. Основы теории ползучести. М.: Наука, 1969. 420 с.

Поступила в редакцию $13 / \mathrm{XII} / 2014$; в окончательном варианте - $11 / \mathrm{II} / 2015$; принята в печать - 08/IV/2015.

Vestn. Samar. Gos. Techn. Un-ta. Ser. Fiz.-mat. nauki

[J. Samara State Tech. Univ., Ser. Phys. \& Math. Sci.], 2015, vol. 19, no. 2, pp. 358-381

ISSN: 2310-7081 (online), 1991-8615 (print)

doi: http://dx.doi.org/10.14498/vsgtu1432

MSC: 74R10, 74G70, 74R15, 74A45, 74R20

\title{
MIXED-MODE LOADING OF THE STRUCTURAL ELEMENTS WITH DEFECT*
}

\author{
L. V. Stepanova, E. M. Yakovleva \\ Samara State University, \\ 1, Academician Pavlov st., Samara, 443011, Russian Federation.
}

\begin{abstract}
In the article the problem of determining the stress-strain state near the mixed-mode crack tip in a power-law material under plane stress conditions is considered. The eigenfunction method is used for the mixed-mode crack tip problem. It is shown that the eigenfunction expansion method results in the nonlinear eigenvalue problem. The numeric solution of the nonlinear eigenvalue problem formulated is obtained. The power of the distance from the crack tip is the eigenvalue of the nonlinear eigenvalue problem considered whereas the angular distributions of the stress components are the
\end{abstract}

(C) 2015 Samara State Technical University.

Please cite this article in press as:

Stepanova L. V., Yakovleva E. M. Mixed-mode loading of the structural elements with defect, Vestn. Samar. Gos. Tekhn. Univ., Ser. Fiz.-Mat. Nauki [J. Samara State Tech. Univ., Ser. Phys. \& Math. Sci.], 2015, vol. 19, no. 2, pp. 358-381. doi: 10.14498/vsgtu1432. (In Russian)

\section{Authors Details:}

Larisa V. Stepanova (Dr. Phys. \& Math. Sci.; stepanovalv@samsu.ru; Corresponding Author), Professor, Dept. of Mathematical Modeling in Mechanics.

Ekaterina M. Yakovleva (adylinaem@samsu.ru), Postgraduate Student, Dept. of Mathematical Modeling in Mechanics.

* This paper is an extended version of the paper [1], presented at the Mathematical Physics and Its Applications 2014 Conference. 
eigenfunctions. The new eigenvalues different from the eigenvalues of the Hutchinson-Rice-Rosengren are found. It is shown that the new asymptotic solution can be interpreted as the self-similar intermediate asymptotics of the stress field in the vicinity of the crack tip at distances which are very small compared to the crack length or the size of the specimen and at distances which are large compared to the length of the completely damaged zone. The developed method allows us to construct the geometry of the completely damaged zone in vicinity of the crack tip.

Keywords: nonlinear eigenvalue problem, stress-strain state near the crack tip, mixed-mode loading, mixity parameter, perturbation technique.

doi: http://dx.doi.org/10.14498/vsgtu1432

Acknowledgments. This work has been supported by the Russian Foundation for Basic Research (project no. 13-01-97009-a-Povolzh'e).

\section{ORCID}

Larisa V. Stepanova: http://orcid.org/0000-0002-6693-3132

\section{REFERENCES}

1. Stepanova L. V., Yakovleva E. M. Mixed-mode loading of the structural elements with defect, The 4nd International Conference "Mathematical Physics and its Applications", Book of Abstracts and Conference Materials; ред. I. V. Volovich; V. P. Radchenko. Samara, Samara State Technical Univ., 2014, C. 336-337 (In Russian).

2. Bui H. D. Fracture Mechanics. Inverse Problems and Solutions, Solid Mechanics and Its Applications, vol.139. Netherlands, Springer, 2006, xxviii+382 pp.. doi: 10.1007/ 978-1-4020-4837-1

3. Li J., Recho N. Méthodes asymptotiques en mécanique de la rupture. Paris, Hermes Science Publications, 2002, $262 \mathrm{pp}$.

4. Handbook of Damage Mechanics: Nano to Macro Scale for Materials and Structures, ed. G. Z. Voyiadjis. New York, Springer, 2015, xxviii+1591 pp.. doi:10.1007/ 978-1-4614-5589-9.

5. François D., Pineau A., Zaoui A. Mechanical Behaviour of Materials. Vol. 1: Microand Macroscopic Constitutive Behaviour, Solid Mechanics and Its Applications, vol. 180. Netherlands, Springer, 2013, xvii+662 pp.. doi : 10.1007/978-94-007-2546-1.

6. Sih G. C. Crack tip mechanics based on progressive damage of arrow: Hierarchy of singularities and multiscale segment, Theoretical and Applied Fracture Mechanics, 2009, vol. 51, no. 1, pp. 11-32. doi: 10.1016/j.tafmec.2009.01.007.

7. Sih G. C., Tang X. S. Simultaneity of multiscaling for macro-meso-micro damage model represented by strong singularities, Theoretical and Applied Fracture Mechanics, 2004, vol. 42, no. 3, pp. 199-225. doi: 10.1016/j.tafmec.2004.09.001.

8. Sih G. C., Tang X. S. Weak and strong singularities reflecting multiscale damage: microboundary conditions for free-free, fixed-fixed and free-fixed constraints, Theoretical and Applied Fracture Mechanics, 2005, vol.43, no. 1, pp. 5-62. doi: 10.1016/j.tafmec. 2004. 12.002.

9. Argatov I. I. Vvedenie $v$ asimptoticheskoe modelirovanie $v$ mekhanike [Introduction to Asymptotic Modelling in Mechanics]. Saint Petersburg, Politekhnika, 2004, 302 pp. (In Russian)

10. Stepanova L. V. Matematicheskie metody mekhaniki razrusheniia [Mathematical Methods of Fracture Mechanics]. Samara, Samara Univ., 2006, 232 pp. (In Russian)

11. Williams M. L. Stress singularities resulting from various boundary conditions in angular corners of plates in extensions, Journal of Applied Mechanics, 1952, vol. 19, pp. 526-534.

12. Williams M. L. On the stress distribution at the base of a stationary crack, Journal of Applied Mechanics, 1957, vol. 24, pp. 109-114. 
13. Beliakova T. A. The eigenspectrum approach and T-stress at the mixed-mode crack tip for a stress-state dependent material, Procedia Materials Sience, 2014, vol. 3, pp. 147-152. doi : 10.1016/j.mspro.2014.06.027.

14. Hutchinson J. W. Singular behaviour at the end of a tensile crack in a hardening material, Journal of the Mechanics and Physics of Solids, 1968, vol.16, no. 1, pp. 13-31. doi: 10. 1016/0022-5096 (68) 90014-8.

15. Hutchinson J. W. Plastic stress and strain fields at a crack tip, Journal of the Mechanics and Physics of Solids, 1968, vol.16, no.5, pp. 337-347. doi:10.1016/0022-5096(68) 90021-5.

16. Rice J. R., Rosengren G. F. Plane strain deformation near a crack tip in a power-law harderning material, Journal of the Mechanics and Physics of Solids, 1968, vol. 16, no. 1, pp. 1-12. doi : 10.1016/0022-5096(68)90013-6.

17. Zehnder A. Fracture Mechanics, Lecture Notes in Applied and Computational Mechanics, vol. 62. Netherlands, Springer, 2012, xiv+226 pp.. doi : 10.1007/978-94-007-2595-9

18. Multiscale Fatigue Crack Initiation and Propagation of Engineering Materials: Structural Integrity and Microstructural Worthiness, Solid Mechanics and its Applications, vol. 152, eds. G. C. Sih. Netherlands, Springer, 2008, xiii+380 pp.. doi: 10.1007/ 978-1-4020-8520-8.

19. Adulina E. M., Igonin S. A., Stepanova L. V. About a non-linear task on eigenvalues incurring from the analysis of tensions at the fatigue crack tip, Vestnik SamGU. Estestvenno-Nauchnaya Ser., 2012, no. 3/1(94), pp. 83-102 (In Russian).

20. Failure and Damage Analises of Advanced Materials, CISM International Centre for Mechanical Sciences, vol.560, eds. H. Altenbach, T. Sadowski. Vienna, Springer, 2015, xii+282 pp.. doi : 10.1007/978-3-7091-1835-1.

21. Hello G., Taha M. B., Roelandt J. M. Analytical determination of coefficients in crack-tip stress expansions for a finite crack in an infinite plane medium, International Journal of Solids and Structures, 2012, vol.49, no. 3-4, pp. 556-566. doi: 10.1016/j.ijsolstr. 2011. 10.024.

22. Shih C. F. Elastic-plastic analysis of combined mode crack problems, PhD Thesis. Harvard University, 1973.

23. Shih C. F. Small-scale yielding analysis of mixed mode plane-strain crack problems, National Symposium on Fracture Mechanics, 1974, vol. STP560, pp. 187-210. doi: 10. $1520 /$ stp33141s.

24. Stepanova L. V., Adylina E. M. Asymptotic methods of nonlinear fracture mechanics: results, contemporary state and perspectives, Vestn. Samar. Gos. Tekhn. Univ. Ser. Fiz.Mat. Nauki [J. Samara State Tech. Univ., Ser. Phys. \& Math. Sci.], 2013, no. 2(31), pp. 156168 (In Russian). doi: 10.14498/vsgtu1156.

25. Shlyannikov V. N., Tumanov A. V. Elastic mode mixity parameters for semi-elliptical crack under biaxial loading, Izv. Saratov Univ. (N.S.), Ser. Math. Mech. Inform., 2010, vol. 10, no. 2, pp. 73-80 (In Russian).

26. Shlyannikov V. N., Kislova S. Yu. Mode mixity parameters for mathematical crack type, Izv. Saratov Univ. (N.S.), Ser. Math. Mech. Inform., 2009, vol.9, no.1, pp. 77-84 (In Russian).

27. Fatigue, Failure, and Damage Evolution, Proceedings of the 2014 Annual Conference on Experimental and Applied Mechanics, vol. 5, eds. J. Carroll, S. Daly. New York, Springer, 2015, viii+252 pp.. doi : 10.1007/978-3-319-06977-7

28. Rahman S., Mohammad E. Effects of mixed-mode overloading on the mixed-mode I+II fatigue crack growth, Archive of Applied Mechanics, 2013, vol.83, no. 7, pp. 987-1000. doi: 10.1007/s00419-013-0731-z.

29. Stepanova L. V. Eigenvalues of the antiplane-shear crack problem for a power-law material, Journal of Applied Mechanics and Technical Physics, 2008, vol.49, no.1, pp. 142-147. doi: $10.1007 / \mathrm{s} 10808-008-0021-7$. 
30. Stepanova L. V. Eigenvalue analysis for a crack in a power-law material, Comput. Math. Math. Phys., 2009, vol.49, no. 8, pp. 1332-1347. doi: 10.1134/S0965542509080053.

31. Stepanova L. V. Refined study of stress-strain state near the crack tip under cyclic loading in a damaged medium, Vestnik SamGU. Estestvenno-Nauchnaya Ser., 2011, no. 2(83), pp. 105-115 (In Russian).

32. Rice J. R. Mathematical analysis in mechanics of fracture, Fracture: An Advanced Treatise, vol. 2, Mathematical Fundamentals; ed. H. Liebowitz. New York, Academic Press, 1968, pp. 191-311, http://esag.harvard.edu/rice/018_Rice_MathAnalMechFract_68.pdf.

33. Stepanova L. V., Yakovleva E. M. Mixed-mode loading of the cracked plate under plane stress conditions, PNRPU Mechanics Bulletin, 2014, no.3, pp. 129-162 (In Russian). doi: 10.15593/perm.mech/2014.3.08.

34. Kachanov L. M. Osnovy teorii polzuchesti [Fundamentals of the theory of creep]. Moscow, Nauka, 1969, 420 pp. (In Russian)

Received 13/XII/2014;

received in revised form $11 / \mathrm{II} / 2015$;

accepted 08/IV/2015. 